\title{
Patient subjective assessment of urinary tract infection frequency and severity is associated with bladder management method in spinal cord injury
}

\author{
Joshua D. Roth $\mathbb{1 0}^{1} \cdot$ Joseph J. Pariser ${ }^{2} \cdot$ John T. Stoffel $^{3} \cdot$ Sara M. Lenherr ${ }^{4} \cdot$ Jeremy B. Myers $^{4}$. \\ Blayne Welk ${ }^{5}$. Sean P. Elliott ${ }^{2}$
}

Received: 5 September 2018 / Revised: 14 February 2019 / Accepted: 15 February 2019 / Published online: 14 March 2019

(c) International Spinal Cord Society 2019

\begin{abstract}
Study design The Neurogenic Bladder Research Group (NBRG) registry is a multicenter prospective observational study. This manuscript is retrospective based on a cross-sectional survey.

Objectives To assess patient subjective assessment of urinary tract infection (UTI) frequency and severity are associated with the degree of use of catheters or incontinence products.

Setting Multiple hospitals across the United States.

Methods Eligibility included: age > 18 years and acquired SCI. Over 1.5 years, 1479 eligible participants were enrolled. We excluded those with surgical reconstruction or diversion of the bladder. In total, 1282 participants were grouped by bladder management: (1) indwelling catheter (IDC), (2) clean intermittent catheterization (CIC), (3) external devices (pads/condom), and (4) volitional voiding (Void). UTI frequency was classified as $0,1-3,4-6$, or $>6$ over the prior year. UTI severity was determined by hospitalization for UTI in the prior year. Multivariate regression compared these factors across groups.

Results UTIs were least frequent in Void followed by pads/condom, CIC, and IDC (all $p \leq 0.001$ ). UTI severity followed a similar pattern. Controlling for covariates, the adjusted odds of UTI frequency (Void = reference) were 2.28 (1.38-3.76) for pads/condom, 3.42 (2.25-5.18) for CIC, and 4.3 (2.59-6.70) for IDC (all $p \leq 0.001)$.

Conclusions Patient subjective assessment of UTI frequency is highest with IDC, followed by CIC, pads/condom, and lowest with spontaneous voiding. The odds of hospitalization for UTI were three times higher for IDC than spontaneous voiding. UTI risk should be considered when counseling patients about bladder management options. These associations do not imply causation but warrant further investigation in a prospective manner.
\end{abstract}

Sponsorship Patient-Centered Outcomes Research Institute (PCORI) Award (CER14092138).

\section{Introduction}

In the United States, $\sim 282,000$ people are living with spinal cord injury (SCI), with an annual incidence of 17,000 cases [1]. Although the etiology of SCI varies with age, the most

Sean P. Elliott

selliott@umn.edu

Indiana University, Indianapolis, IN, USA

2 University of Minnesota, Minneapolis, MN, USA

3 University of Michigan, Ann Arbor, MI, USA

4 University of Utah, Salt Lake City, UT, USA

5 Western University, London, ON, Canada common cause is trauma [1,2]. Although predominantly affecting younger individuals, the average age at injury has increased in recently to 42 years. The majority of those with SCI are male and non-Hispanic white [1].

Historically, renal failure was the leading cause of death in those with SCI [3]. With advances in bladder management and renal replacement therapy, renal failure is now an uncommon cause of death [3]. Urinary tract infection (UTI) is a major source of morbidity with urosepsis as one of the leading causes of death. Despite improved antiinfective catheter materials, prophylactic antibiotics, and antiseptic agents, sepsis from UTI remains a leading cause of death in SCI patients $[1,4,5]$. Pneumonia/respiratory issues represent the leading cause of mortality in this population $[1,4,5]$. Approximately $30 \%$ of those with SCI are hospitalized one or more times annually [1]. UTIs and other genitourinary 
complaints are the leading cause of admissions and the most common cause of health care utilization in this population [6]. Furthermore, UTIs are a significant cause of distress for people with SCI [7].

Owing to a neurogenic bladder from SCI, most individuals require a bladder emptying strategy, which can include (1) indwelling catheter (IDC), (2) clean intermittent catheterization (CIC), (3) external devices (pads, diapers, condom catheter [pads/condom]), or (4) volitional voiding (Void). The selection of the type of bladder management depends on level of SCI and potential impairment in upper extremity motor function, patient gender, patient age, and patient preferences [8]. The main goal of this management is to prevent complications of neurogenic lower urinary tract dysfunction such as kidney failure, urinary tract infections, urinary stones, or urethral injuries, and to avoid urinary incontinence. Current evidence suggests that IDC is associated with a higher risk of UTI than CIC but data are scarce, relying on small sample sizes, and comparisons with external devices or Void are lacking $[9,10]$.

Given the current national focus on reducing catheterassociated UTIs in the non-SCI population, and in light of the particularly high morbidity associated with UTI in the SCI population, we sought to determine the frequency and severity of UTI associated with the degree of use of catheters or other incontinence products. This study was funded by the Patient-Centered Outcomes Research Institute (PCORI), which promotes improvements in patient care through an application of patient-centered outcomes research. The goal of this study was to examine the frequency and severity of UTIs from a patient perspective, as opposed to culture proven UTIs so as to capture patientcentered outcomes. Although these patient-centered outcomes may not represent true UTIs, they do represent morbidity of disease and often consume health care resources, whereas impacting quality of life in our patients. Our hypothesis is that frequency and severity of UTIs correlate with the degree of catheter exposure; that is, UTI frequency/severity are lowest the Void group, followed by pads/condom, followed by CIC and finally the highest risk in IDC.

\section{Methods}

The Neurogenic Bladder Research Group (NBRG) registry is a multicenter prospective observational study of people with SCI; the primary objective is to compare neurogenic bladder related quality of life across different methods of bladder management (clinicaltrials.gov NCT02616081). In accordance with the goals of studies funded by the Patient Centered Outcomes Research Institute, study design, execution, interpretation of findings and dissemination of conclusions were all conducted with significant input from stakeholders with SCI.

Registry eligibility included: age $>18$ years, ability to effectively communicate in written and spoken English and acquired SCI (including, e.g., traumatic, spinal cord stroke, tumor without current malignancy, surgical injury, and transverse myelitis). We excluded people with congenital SCI (e.g., cerebral palsy, spina bifida, caudal regression, or sacral agenesis) and people with progressive SCI (e.g., active malignancy or progressive neurologic diseases affecting the spinal cord such as multiple sclerosis). Participants were recruited from urology clinics and rehabilitation clinics at the Universities of Michigan, Minnesota, and Utah, inpatient rehabilitation centers, nursing homes and through online advertisement on social media websites and SCI advocacy websites. Institutional Review Board approval was obtained at each university site.

Participants were enrolled over a 1.5-year period. Participants completed an enrollment interview with a trained study coordinator. The enrollment interview included standardized questions that documented demographic and clinical data relevant to SCI and neurogenic bladder. Given the community-based study design, no medical chart review was performed.

Although, the principal aim was to compare patientreported outcomes through standardized, validated assessments prospetively, we also collected adjunct data at enrollment about conditions that would impact upon quality of life, such as the self-reported frequency and severity of UTIs. For the purposes of the current manuscript, we conducted a retrospective review focused on responses to two questions in the enrollment questionnaire delivered at enrollment that assessed patient-perceived UTI frequency and severity over the 12 months prior to enrollment: (1) "How many urinary tract infections (including bladder or kidney infections) have you had over the past 12 months?" which was reported as a categorical variable $(0,1-3,4-6$, $>6$ in the last year) and (2) "Have you been hospitalized for a UTI or kidney infection in the last 12 months?" which was reported as a binary variable (yes, no). This paper aims to assess the frequency and severity of patient-perceived UTIs, which may not represent what physicians consider to be a culture proven "UTI," but can cause significant distress in patients with SCI [7].

For this study, we excluded registry participants with surgical reconstruction or diversion of the urinary bladder and grouped the remaining participants by their primary bladder management as follows: (1) IDC, (2) CIC, (3) external devices (pads, diapers, condom catheter [pads/ condom]), and (4) Void. Void was determined by asking patients "Do you control your urination normally and urinate into a toilet?" Baseline demographic and clinical data were compared across bladder management groups using 
chi squared tests for categorical variables and analysis of variance for continuous variables. UTI frequency and hospitalization were compared across groups and analyzed using chi-squared tests. Next, univariate logistic regression was performed to examine risk factors for UTI frequency and hospitalization. Multivariate ordinal regression was then performed to determine risk factors for UTI frequency and hospitalization while controlling for confounders. Possible confounders included age, gender, body mass index, income level, in-home support, age-adjusted Charlson comorbidity index (CCI), level of injury, and duration since injury. Age-adjusted CCI is a measure of disease burden arising from multiple comorbidities, with higher scores representing sicker patients [11]. Income level and in-home support were included as surrogates for the amount of support available to patients. $P<0.05$ was considered statistically significant.

\section{Results}

A total of 1479 people were enrolled in the study; 1282 participants had no prior bladder reconstruction and qualified for the current investigation. Bladder management groups included CIC $(n=753)$, IDC $(n=271)$, pads/condom $(n=150)$, and Void $(n=109)$. In those who performed CIC, the mean number of daily catheterizations was 5.94 (SD 1.81). Baseline characteristics are described in Table 1. Most participants were men, and there was little difference by bladder management group; however, within the pads/condom group it should be noted that only men used a condom catheter and that women represented a majority of the pad/diaper group. Those who performed CIC were younger (43.7 vs. > 45.9) and had a lower BMI (26.1 vs. $>27.2$ ) than those in the other groups. Level of SCI (\% tetraplegia) was lowest in CIC (30\%) followed by Void (43\%), pads/condom (51\%) and IDC (69\%).

The overall incidence of UTI in the present study is comparable to an incidence rate of $0.3-1$ UTIs per 100 person-days. UTIs were least frequent in Void followed by pads/condom, CIC and IDC $(p<0.001$; Fig. 1). UTI severity followed a similar pattern: Void $(6 \%$ hospitalization) followed by pads/condom (9\%), CIC (11\%), and IDC (22\%; $p<0.001)$ (Table 2). We also performed an analysis of UTI frequency and severity between patients with an indwelling urethra catheter $(N=190)$ compared with a suprapubic tube $(N=81)$, and there was no significant difference ( $p=0.171$ and $p=0.323$, respectively).

Using multivariate ordinal regression analysis, models were constructed to examine the impact of bladder management strategy on UTI rates and severity while controlling for demographic and clinical factors (Table 3). The adjusted odds of increased UTI frequency (reference: Void) were $2.28(1.38-3.76, p=0.001)$ for pads/condom, 3.42 (2.25-5.18, $p<0.001)$ for CIC and $4.12(2.59-6.70, p<$ $0.001)$ for IDC. Other factors independently associated with rate of UTIs were younger age $(\mathrm{OR}=1.02$ (1.01-1.04), $<0.001$ ) for each additional year, $p<0.001)$, female gender $(\mathrm{OR}=1.42(1.13-1.79), p=0.002)$, and in-home support of "Hired Help" $(\mathrm{OR}=1.76(1.32-2.36), p=0.001)$ and "Other" $(\mathrm{OR}=2.25(1.51-3.35), p<0.001)$. The odds of UTI hospitalization (Void $=$ reference) were significantly elevated only for IDC (2.9 (1.07-7.85), $p=0.036)$. Ageadjusted CCI of $2+(\mathrm{OR}=2.45(1.18-5.09), p=0.017)$ and more in-home support of "Hired Help" $(\mathrm{OR}=2.20$ (1.30-3.70), $p=0.003$ ) was associated with higher odds of increased UTI hospitalization. Higher household income (\$40,000-75,000/year compared with $<\$ 40,000 /$ year) was associated with decreased UTI hospitalization $(\mathrm{OR}=0.56$ (0.32-0.99), $p=0.047)$.

When stratifying our analysis by gender, women were noted to have more UTIs than men (OR 1.42, $p=0.002$ ), however, there was no difference in rates of hospitalization ( $p=0.136)$. For men, compared with spontaneous voiding, IDC (OR 7.04, $p<0.001$ ), CIC (OR 4.68, $p<0.001$ ), and condom catheter (OR 3.68, $p=0.001$ ) were all associated with higher rates of UTI. For women, compared with spontaneous voiding, IDC (OR 2.18, $p=0.04$ ) and CIC (OR 2.38, $p=0.01$ ) were associated with higher rates of UTI, whereas pads were not $(p=0.75)$.

For our model, the determination coefficient, $R^{2}$, is less than or equal to 0.1 , meaning that $10 \%$ of the dependent variable is predictable by the independent variables. Further, the model's goodness of fit, as measured by the cstatistic, is greater than or equal to 0.635 , indicated the model fit is acceptable.

\section{Discussion}

The present study is the largest to compare incidence of patients' subjective appreciation of UTIs across all of these management strategies. We found the risk of UTI is highest with IDC, followed by CIC, then pads/condom, and lowest with spontaneous voiding. This association remained significant even when controlling for multiple demographic and clinical variables. A subset analysis of the IDC group showed that there was no significant difference in UTI rate or hospitalization between patients with indwelling urethral catheter vs. suprapubic tube. Previous studies have shown no difference in UTI incidence between CIC and condom drainage [12], between CIC and voiding [13], or between CIC, condom drainage and voiding [14]. In addition, current studies have small sample sizes, which could make the data unreliable [12, 13]. For example, in the Escarlin De Ruz et al. paper, there are only 41 patients using a condom 
Table 1 Baseline patient characteristics based on primary bladder management

\begin{tabular}{|c|c|c|c|c|c|}
\hline & \multicolumn{4}{|c|}{ Primary bladder management group } & \multirow[t]{2}{*}{$P$ value } \\
\hline & Spontaneous Void to toilet & Pads/condom & CIC & Indwelling catheter & \\
\hline Number & 109 & 150 & 753 & 271 & \\
\hline Age mean $(\mathrm{SD})$ & $47.7(13.1)$ & $48.7(12.8)$ & $43.7(13.1)$ & $45.9(13.2)$ & $<0.001$ \\
\hline Gender $N(\%)$ & & & & & 0.223 \\
\hline Male & $68(62.4)$ & $94(62.7)$ & $504(66.9)$ & $164(60.5)$ & \\
\hline Female & $41(37.6)$ & $56(37.3)$ & $248(32.9)$ & $107(39.5)$ & \\
\hline BMI mean (SD) & $27.9(6.9)$ & $27.4(7.6)$ & $26.1(6.0)$ & $27.2(7.8)$ & 0.005 \\
\hline Income level & & & & & 0.007 \\
\hline$<40 \mathrm{~K}$ & $31(28.4)$ & $52(34.7)$ & $263(34.9)$ & $123(45.4)$ & \\
\hline $40-75 \mathrm{~K}$ & $26(23.9)$ & $27(18.0)$ & $154(20.5)$ & $48(17.7)$ & \\
\hline$>75 \mathrm{~K}$ & $19(17.4)$ & 25 (16.7) & $163(21.6)$ & $38(14.0)$ & \\
\hline Unknown & $32(29.4)$ & $45(30.0)$ & $169(22.4)$ & $57(21.0)$ & \\
\hline In-home support & & & & & $<0.001$ \\
\hline None & $57(52.3)$ & $61(40.7)$ & $380(50.5)$ & $40(14.8)$ & \\
\hline Hired help & $14(12.8)$ & $46(30.7)$ & $160(21.3)$ & $140(51.7)$ & \\
\hline Live in family/friend & $26(23.9)$ & $24(16.0)$ & $137(18.2)$ & 49 (18.1) & \\
\hline Other & $10(9.2)$ & $13(8.7)$ & $66(8.8)$ & $16(5.9)$ & \\
\hline Missing & $2(1.8)$ & $6(4.0)$ & $10(1.3)$ & $26(9.6)$ & \\
\hline Age-adjusted CCI & & & & & $<0.001$ \\
\hline 0 & $57(52.3)$ & $65(43.3)$ & $458(60.8)$ & $141(52.0)$ & \\
\hline 1 & $23(21.1)$ & $49(32.7)$ & $174(23.1)$ & $64(23.6)$ & \\
\hline $2+$ & $29(26.6)$ & $36(24.0)$ & $121(16.1)$ & $66(24.4)$ & \\
\hline Level of injury & & & & & $<0.001$ \\
\hline Tetraplegic & $47(43.1)$ & $77(51.3)$ & $229(30.4)$ & $188(69.4)$ & \\
\hline Paraplegic & $58(53.1)$ & $66(44)$ & $514(68.2)$ & $80(29.5)$ & \\
\hline Unknown & $4(3.7)$ & $5(3.3)$ & $9(1.2)$ & $3(1.1)$ & \\
\hline Duration since injury mean (SD) & $9.5(10.8)$ & $16.0(12.9)$ & $12.5(10.4)$ & $15.6(12.7)$ & $<0.001$ \\
\hline
\end{tabular}

Numbers may not add up to $100 \%$ owing to missing data

Pads/condom includes spontaneoulsy voiding to diapers, spontaneously voiding to pads, and condom catheter

Fig. 1 Frequency of UTIs in prior year

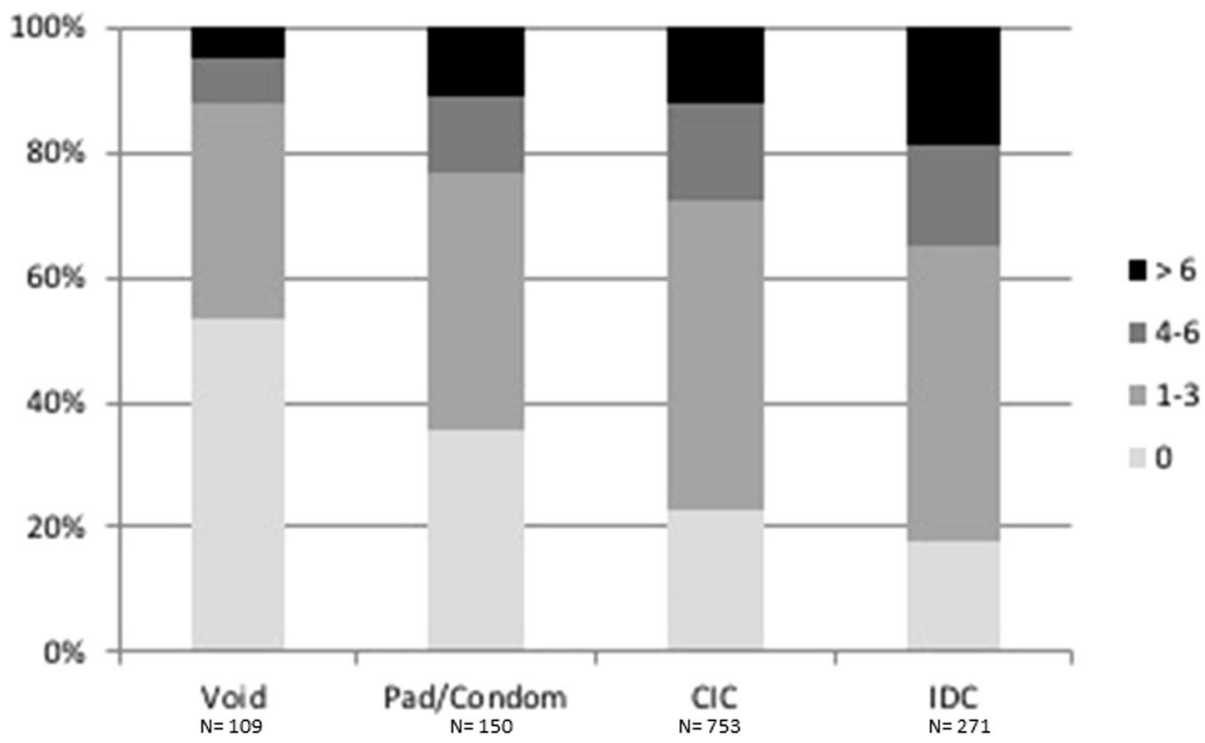


Table 2 UTI rate and UTI hospitalization based on bladder management
Primary bladder management group

\begin{tabular}{lcccc}
\hline \multicolumn{5}{c}{ Primary bladder management group } \\
\cline { 2 - 5 } & Spontaneous Void to toilet & Pads/condom & CIC & Indwelling catheter \\
\hline Number & 109 & 150 & 753 & 271 \\
UTI in the last year $N(\%)$ & & & \\
0 & $58(53.2)$ & $53(35.3)$ & $172(22.8)$ & $49(18.1)$ \\
$1-3$ & $37(33.9)$ & $62(41.3)$ & $372(49.4)$ & $126(46.5)$ \\
$4-6$ & $8(7.3)$ & $18(12.0)$ & $117(15.5)$ & $44(16.2)$ \\
$>6$ & $5(4.6)$ & $17(11.3)$ & $92(12.2)$ & $52(19.2)$ \\
UTI hospitalization in the last year $N(\%)$ & & & \\
Yes & $6(5.5)$ & $13(8.7)$ & $82(10.9)$ & $59(21.8)$ \\
No & $103(94.5)$ & $137(91.3)$ & $671(89.1)$ & $212(78.2)$ \\
\hline
\end{tabular}

Numbers may not add up to $100 \%$ owing to missing data

\begin{tabular}{|c|c|c|c|c|c|c|}
\hline & \multicolumn{3}{|l|}{ UTI rate } & \multicolumn{3}{|c|}{ UTI hospitalization } \\
\hline & Adjusted OR & $95 \% \mathrm{CI}$ & $p$ & Adjusted OR & $95 \% \mathrm{CI}$ & $p$ \\
\hline \multicolumn{7}{|c|}{ Bladder management group (ref: Void) } \\
\hline Pads/condom & 2.28 & $1.38-3.76$ & 0.001 & 1.32 & $0.43-4.03$ & 0.627 \\
\hline Clean intermittent catheter & 3.42 & $2.25-5.18$ & $<0.001$ & 2.06 & $0.80-5.31$ & 0.137 \\
\hline Indwelling catheter & 4.16 & $2.59-6.70$ & $<0.001$ & 2.90 & $1.07-7.85$ & 0.036 \\
\hline Age (per year increase) & 1.02 & $1.01-1.04$ & $<0.001$ & 1.02 & $1.00-1.04$ & 0.060 \\
\hline Female gender (ref: male) & 1.42 & $1.13-1.79$ & 0.002 & 0.73 & $0.48-1.10$ & 0.136 \\
\hline BMI (per kg/m² increase) & 1.00 & $0.98-1.02$ & 0.841 & 1.00 & $0.97-1.03$ & 0.872 \\
\hline \multicolumn{7}{|l|}{ Age-adjusted CCI (ref: 0) } \\
\hline 1 & 1.17 & $0.84-1.63$ & 0.366 & 1.39 & $0.77-2.52$ & 0.270 \\
\hline $2+$ & 1.41 & $0.91-2.21$ & 0.129 & 2.45 & $1.18-5.09$ & 0.017 \\
\hline \multicolumn{7}{|c|}{ Household Income (ref $<\$ 40 \mathrm{~K} / \mathrm{yr})$} \\
\hline$\$ 40-75 \mathrm{~K}$ & 0.92 & $0.68-1.25$ & 0.589 & 0.56 & $0.32-0.99$ & 0.047 \\
\hline$>\$ 75 \mathrm{~K}$ & 0.78 & $0.58-1.07$ & 0.121 & 0.57 & $0.32-1.01$ & 0.052 \\
\hline \multicolumn{7}{|l|}{ In-home support (ref: none) } \\
\hline Hired help & 1.76 & $1.32-2.36$ & 0.001 & 2.20 & $1.30-3.70$ & 0.003 \\
\hline Live in family/friend & 1.35 & $0.99-1.83$ & 0.058 & 1.73 & $0.98-3.06$ & 0.059 \\
\hline Other & 2.25 & $1.51-3.35$ & $<0.001$ & 2.48 & $1.27-4.87$ & 0.008 \\
\hline \multicolumn{7}{|l|}{ Level of injury (ref: tetraplegia) } \\
\hline Paraplegia & 1.11 & $0.86-1.42$ & 0.422 & 0.84 & $0.55-1.30$ & 0.434 \\
\hline Unknown & 0.97 & $0.38-2.46$ & 0.943 & 0.54 & $0.07-4.36$ & 0.562 \\
\hline Duration since Injury (per year) & 1.00 & $0.99-1.01$ & 0.555 & 1.01 & $1.00-1.03$ & 0.137 \\
\hline
\end{tabular}

Table 3 Multivariate ordinal regression for UTI rate and UTI hospitalization catheter and 25 patients with normal voiding. Furthermore, this study was not a very accurate representation of UTI rates because all patients underwent a surveillance urine culture every 10 days regardless of symptoms [12]. Although patients were only considered to have a UTI if the culture was positive and they had symptoms; there is significant bias involved in checking urine cultures every 10 days.

Although those with pads/condoms have constant exposure to products, this exposure is external, which may decrease the risk of UTI as opposed to internal products like CIC or IDC. In this study, all patients who used condom catheters were men and pads users were disproportionately women. Whereas women tended to have more UTIs, the effect of product exposure on UTIs was greater in men.

UTI is a major source of morbidity in SCI patients [1, 2, 14-21]. Incomplete voiding, elevated intravesical pressures, and catheter use all contribute to an increased risk of symptomatic UTI [19]. Although patients with SCI are often recommended to manage their bladder with $\mathrm{CIC}$ or 
IDC, there remains a relative lack of data on the rate of UTIs across management strategies. The current study represents the largest evaluation of patient subjective assessment of UTI incidence and severity in the management of neurogenic bladder owing to SCI.

Previous research has shown that long-term IDC is associated with reduced bladder compliance (i.e., reduced elasticity and increased bladder pressures) and reduced compliance results in an increased likelihood of bacterial invasion across the mucosa of the bladder [17, 22]. Similarly, bladder ischemia, triggered by increased bladder pressure from low bladder compliance, bladder outflow obstruction or overdistension influences the presence of UTIs [23]. In other words, reduced bladder compliance is one factor in the progression from bacterial colonization to bacterial infection.

In all, $188 / 541(35 \%)$ of participants with tetraplegia were managed with IDC, compared with only 80/718 (11\%) of participants with paraplegia. Cameron et al. [24] have shown that the prevalence of IDC increases from $23 \%$ in the first year after SCI to $45 \% 30$ years after injury. Because IDC is highly associated with increased frequency of UTIs and hospitalization for UTIs, and because people with tetraplegia are most likely to be managed with IDC, we should pursue strategies that enable people with tetraplegia to avoid IDC. Adaptive technology such as tenodesis splints can augment upper extremity function and facilitate CIC in people with partial upper extremity function. Upper arm surgeries, such as tendon and nerve transfers, are also an option. Surgical creation of a continent catheterizable stoma on the abdominal wall (e.g., Mitrofanoff) facilitates ease of catheterization.

The overall patient subjective assessment of UTI incidence in the present study is comparable to an incidence rate of $0.3-1$ UTIs per 100 person-days, which is in line with the reported incidence rate of 0.64-0.68 UTIs per hundred person-days in the literature [12,14]. Although this does not imply that self-reported measures of UTIs provide valid estimates of incidence rates, the fact that our reported rates are similar to those reported in the literature suggests that our measure approximates the real incidence of UTIs in this population. UTIs occurred most frequently in those with IDC in the present study, which is similar to other series [12-14]. IDCs are the strongest risk factor for UTI in SCI patients [16]. The risk of UTI increases with increasing duration of IDC [25]. This is owing to an adherent bacterial biofilm, which ascends through the luminal and external surfaces of an IDC within 8-24 h. This leads to bacterial adherence on the bladder surface and symptomatic UTI [17]. owing to chronic bacterial colonization within biofilms, even targeted antibiotic treatment may fail to eradicate the catheter-associated UTI, and routine IDC changes may need to be scheduled every 1-2 weeks [16].
UTI severity followed a similar pattern to UTI incidence: IDC (22\% hospitalization), CIC (11\%), pads/condom (9\%), and Void (6\%). However, IDC remained the only bladder management strategy with increased odds of hospitalization compared to voiding when controlling for covariates. Owing to the risk of sepsis and death, suspected UTIs should be thoroughly evaluated and treated appropriately. In addition, UTIs account for up to $40 \%$ of nosocomial infections, many being catheter related $[9,16,19,25]$. UTIs accounted for $70 \%$ of fevers in SCI patients admitted to a rehabilitation unit and was shown to significantly prolong admission. In patients with fever, the use of an IDC was significantly higher compared with CIC and spontaneous voiding [26]. Early infection after SCI may affect long-term neurologic recovery, making the prevention of febrile infection a high priority [27]. A study analyzing predictors of mortality in veterans with SCI revealed $40 \%$ of patients were deceased at the end of a 12-year study period, with UTI being the third leading cause of death (14\%) behind pneumonia (21\%) and cancer (16\%) [4]. Further, this study revealed that those with hired in home help were more likely to have UTIs, which is likely a marker for decreased functional status. This corroborates previous reports of decreased functional independence and those requiring maximal assistance as risk factors for UTIs [20].

In some ways, this analysis supports the existing literature. We show that UTI frequency and severity are higher in IDC than CIC; the higher risk of UTI is one of the many reasons physicians often recommend CIC over IDC [28, 29]. However, in other ways, our findings challenge existing dogma. Guidelines recommend caution when managing patients with Crede voiding and Valsalva voiding because emptying can occur at high pressure, can be incomplete, associated with urinary tract infections, and can cause vesicoureteral reflux owing to increased bladder pressure $[28,29]$. We do not know the voiding characteristics of our pads/condom group; specifically, we do not know how many void by Valsalva vs. Crede vs. have frequent incontinence owing to detrusor overactivity. Still, we demonstrate that participants managed with pads/condom report fewer UTIs than participants on CIC. These data should not be interpreted to recommend against CIC; rather, these data suggest that from a UTI perspective it may be safe to allow some individuals with SCI to utilize pads/condom (where conventionally, they would be recommended to perform CIC or have an IDC). As this refutes existing treatment recommendations, further research is warranted to identify which patients would benefit from CIC and which would be safe to utilize pads/condom.

Our study has several limitations. First, this study relied on self-reported UTIs, rather than culture-confirmed UTIs. This may overestimate the number of UTIs as self-reported UTI in individuals with SCI has been shown to have a positive 
predictive value of only $33 \%[30,31]$. However, this study measures the morbidity associated with UTIs, which correlates with health care utilization and cost [32]. Further, although selfreported rates of UTIs may not be accurate, the degree of inaccuracy should not differ across bladder management groups. The patients were not randomized, and their method of bladder management was determined based on their clinical situation. Further, each patient was categorized into a bladder management group based on the method they used for the majority of time; yet, multiple bladder management methods could have been used in any given patient. It would have been impractical to have more than four categories for the purpose of analysis, this would make numbers too small to power, so patients were grouped by the method they used for the majority of the time. In addition, there were several items we did not control for, such as menopausal status in women, comorbidities such as diabetes, intradetrusor botulinum toxin usage, anticholinergic usage, or urodynamic data that could be investigated with future studies. However, the analyzing the use of botulinum toxin and anticholinergics in this population without knowing urodynamic parameters would risk misattribution bias. The data were also collected retrospectively, and as such this work should be viewed as exploratory. Further investigation with a prospective cohort study using a representative sample in which a prespecified definition of UTI is used that follows the NIDRR definition and includes both signs and symptoms of a UTI as well as culture proven UTI should aim to more rigorously prove a causal link between method of bladder emptying and UTIs [33].

In conclusion, UTI frequency is highest with IDC, followed by CIC, then pads/condom, and lowest with spontaneous voiding. The odds of hospitalization for UTI were three times higher for IDC than for spontaneous voiding. UTI risk should be considered when counseling patients about bladder management options. These associations do not imply causation but warrant further investigation in a prospective manner.

\section{Data archiving}

The data sets generated and/or analyzed during the current study are available from the corresponding author on reasonable request.

Author contributions JDR was responsible for the interpretation of results and manuscript writing. JJP was responsible for data analysis and manuscript revision. JTS, SML, JBM, BW and SPE aided in study design, acquired data, and revised the manuscript. All authors approved the final version of the manuscript.

\section{Compliance with ethical standards}

Conflict of interest The authors declare that they have no conflict of interest.
Statement of ethics I/we certify that all applicable institutional and governmental regulations concerning the ethical use of human volunteers/animals were followed during the course of this research.

Publisher's note: Springer Nature remains neutral with regard to jurisdictional claims in published maps and institutional affiliations.

\section{References}

1. Spinal Cord Injury (SCI). 2016 facts and figures at a glance. J Spinal Cord Med. 2016;39:493-4.

2. Ge L, Arul K, Ikpeze T, Baldwin A, Nickels JL, Mesfin A. Demographics and clinical characteristics of traumatic and nontraumatic spinal cord injuries. World Neurosurg. 2017. https://doi. org/10.1016/j.wneu.2017.12.008.

3. Strauss DJ, DeVivo MJ, Paculdo DR, Shavelle RM. Trends in life expectancy after spinal cord injury. Arch Phys Med Rehabil. 2006;87:1079-85.

4. Rabadi MH, Mayanna SK, Vincent AS. Predictors of mortality in veterans with traumatic spinal cord injury. Spinal Cord. 2013;51:784-8.

5. Frankel HL, Coll JR, Charlifue SW, Whiteneck GG, Gardner BP, Jamous MA, et al. Long-term survival in spinal cord injury: a fifty year investigation. Spinal Cord. 1998;36:266-74.

6. Skelton F, Hoffman JM, Reyes M, Burns SP. Examining healthcare utilization in the first year following spinal cord injury. $\mathrm{J}$ Spinal Cord Med. 2015;38:690-5.

7. Craven C, Hitzig SL, Mittmann N. Impact of impairment and secondary health conditions on health preference among Canadians with chronic spinal cord injury. J Spinal Cord Med. 2012;35:361-70.

8. Zlatev DV, Shem K, Elliott CS. Predictors of long-term bladder management in spinal cord injury patients-Upper extremity function may matter most. Neurourol Urodyn. 2017;36:170.

9. Hooton TM, Bradley SF, Cardenas DD, Colgan R, Geerlings SE, Rice JC, et al. Diagnosis, prevention, and treatment of catheterassociated urinary tract infection in adults: 2009 International Clinical Practice Guidelines from the Infectious Diseases Society of America. Clin Infect Dis. 2010;50:625-63.

10. Cameron AP, Wallner LP, Forchheimer MB, Clemens JQ, Dunn RL, Rodriguez G, et al. Medical and psychosocial complications associated with method of bladder management after traumatic spinal cord injury. Arch Phys Med Rehabil. 2011;92:449-56.

11. Charlson ME, Pompei P, Ales KL, MacKenzie CR. A new method of classifying prognostic comorbidity in longitudinal studies: development and validation. J Chronic Dis. 1987;40:373-83.

12. Esclarín De Ruz A, García Leoni E, Herruzo Cabrera R. Epidemiology and risk factors for urinary tract infection in patients with spinal cord injury. J Urol. 2000;164:1285-9.

13. Erickson RP, Merritt JL, Opitz JL, Ilstrup DM. Bacteriuria during follow-up in patients with spinal cord injury: I. Rates of bacteriuria in various bladder-emptying methods. Arch Phys Med Rehabil. 1982;63:409-12.

14. Singh R, Rohilla RK, Sangwan K, Siwach R, Magu NK, Sangwan SS. Bladder management methods and urological complications in spinal cord injury patients. Indian J Orthop. 2011;45:141-7.

15. Biering-Sørensen F, Bagi P, Høiby N. Urinary tract infections in patients with spinal cord lesions: treatment and prevention. Drugs. 2001;61:1275-87.

16. Biering-Sørensen F. Urinary tract infection in individuals with spinal cord lesion. Curr Opin Urol. 2002;12:45-49.

17. Siroky MB. Pathogenesis of bacteriuria and infection in the spinal cord injured patient. Am J Med. 2002;113:67S-79S. 
18. Trautner BW, Darouiche RO. Prevention of urinary tract infection in patients with spinal cord injury. J Spinal Cord Med. 2002;25:277-83.

19. García Leoni ME, Esclarín De, Ruz A. Management of urinary tract infection in patients with spinal cord injuries. Clin Microbiol Infect. 2003;9:780-5.

20. D'Hondt F, Everaert K. Urinary tract infections in patients with spinal cord injuries. Curr Infect Dis Rep. 2011;13:544-51.

21. Skelton F, Grigoryan L, Holmes SA, Poon IO, Trautner B. Routine urine testing at 436 the spinal cord injury annual evaluation leads to unnecessary antibiotic use: a 437 pilot study and future directions. Arch Phys Med Rehabil. 2018;99:219-25.

22. Weld KJ, Graney MJ, Dmochowski RR. Differences in bladder compliance with time and associations of bladder management with compliance in spinal cord injured patients. J Urol. 2000;163:1228-33.

23. Vasudeva P, Madersbacher H. Factors implicated in pathogenesis of urinary tract infections in neurogenic bladders: some revered, few forgotten, others ignored. Neurourol Urodyn. 2013;33:95-100.

24. Cameron AP, Wallner LP, Tate DG, Sarma AV, Rodriguez GM, Clemens JQ. Bladder management after spinal cord injury in the United States 1972 to 2005. J Urol. 2010;184:213-7.

25. Foxman B. Epidemiology of urinary tract infections: incidence, morbidity, and economic costs. Dis Mon. 2003;49:53-70.

26. Unsal-Delialioglu S, Kaya K, Sahin-Onat S, Kulakli F, Culha C, Ozel S. Fever during rehabilitation in patients with traumatic spinal cord injury: analysis of 392 cases from a national rehabilitation hospital in Turkey. J Spinal Cord Med. 2010; 33:243-8.

27. Failli V, Kopp MA, Gericke C, Martus P, Klingbeil S, Brommer $\mathrm{B}$, et al. Functional neurological recovery after spinal cord injury is impaired in patients with infections. Brain. 2012; 135:3238-50.

28. Consortium for Spinal Cord Medicine. Bladder management for adults with spinal cord injury: a clinical practice guideline for health-care providers. J Spinal Cord Med. 2006;29:527-73.

29. Urologic Management of the Spinal Cord Injured Patient. 2016.

30. Ronco E, Denys P, Bernède-Bauduin C, Laffont I, Martel P, Salomon J, et al. Diagnostic criteria of urinary tract infection in male patients with spinal cord injury. Neurorehabil Neural Repair. 2011;25:351-8.

31. Massa LM, Hoffman JM, Cardenas DD. Validity, accuracy, and predictive value of urinary tract infection signs and symptoms in individuals with spinal cord injury on intermittent catheterization. J Spinal Cord Med. 2009;32:568-73.

32. Foxman B, Barlow R, D'Arcy H, Gillespie B, Sobel JD. Urinary tract infection: self-reported incidence and associated costs. Ann Epidemiol. 2000;10:509-15.

33. The prevention and management of urinary tract infections among people with spinal cord injuries. National Institute on Disability and Rehabilitation Research Consensus Statement. January 27-29, 1992. J Am Paraplegia Soc. 1992;15:194-204. 